Title: A bioimpedance spectroscopy-based method for diagnosis of lower-limb lymphedema

Running title: Diagnosing lower-limb lymphedema using BIS

Authors: Megan L Steele $\mathrm{PhD}^{1,2}$, Monika Janda $\mathrm{PhD}^{1,3}$, Dimitrios Vagenas $\mathrm{PhD}^{1}$, Leigh $\mathrm{C}$ Ward $\mathrm{PhD}^{4}$, Bruce H Cornish $\mathrm{PhD}^{1}$, Robyn Box $\mathrm{PhD}^{5}$, Susan Gordon $\mathrm{PhD}^{6,7}$, Melanie Matthews ${ }^{6}$, Sally D Poppitt $\mathrm{PhD}^{8}$, Lindsay D Plank $\mathrm{PhD}^{9}$, Wilson Yip MSc${ }^{8}$, Angela Rowan ${ }^{10}$, Hildegard Reul-Hirche DipPhty ${ }^{11}$, Andreas Obermair MD ${ }^{12}$ and Sandra C Hayes $\mathrm{PhD}^{1}$

\title{
Institutions:
}

1. Institute of Health and Biomedical Innovation, Queensland University of Technology, Brisbane, Queensland, Australia.

2. School of Clinical Medicine, The University of Queensland, Brisbane, Queensland, Australia.

3. Centre for Health Services Research, Faculty of Health, The University of Queensland, Brisbane, Queensland, Australia.

4. School of Chemistry and Molecular Biosciences, The University of Queensland, Brisbane, Queensland, Australia.

5. Queensland Lymphedema \& Breast Oncology Physiotherapy, Brisbane, Queensland, Australia.

6. College of Healthcare Sciences, James Cook University, Townsville, Queensland, Australia.

7. College of Nursing and Health Sciences, Flinders University, Adelaide, South Australia. 
8. Human Nutrition Unit and School of Biological Sciences, University of Auckland, Auckland, New Zealand.

9. Department of Surgery, University of Auckland, Auckland, New Zealand.

10. Fonterra Co-operative Group Limited, Auckland, New Zealand.

11. Royal Brisbane and Women's Hospital, Physiotherapy Department, Brisbane, Queensland, Australia.

12. Queensland Centre for Gynaecological Cancer Research, The University of Queensland, Brisbane, Queensland, Australia.

Corresponding author: Dr Sandra C Hayes

Address: 60 Musk Avenue, Institute of Health and Biomedical Innovation, Queensland University of Technology, Kelvin Grove, Queensland, 4059, Australia

Email: sc.hayes@qut.edu.au

Phone: +971524964248

Keywords: lymphedema, bilateral, unilateral, lower-limb, diagnosis, upper-limb 


\begin{abstract}
BACKGROUND: This study aimed to test whether arm-to-leg ratios of extracellular water (ECW) and ECW normalised to intracellular water (ICW), measured by bioimpedance spectroscopy (BIS), can accurately detect bilateral, lower-limb lymphedema, and whether accounting for sex, age and body mass index (BMI) improves the diagnostic performance of cut-offs.
\end{abstract}

METHODS AND RESULTS: We conducted a dual approach, case-control study consisting of cases of bilateral, lower-limb lymphedema and healthy controls who self-reported absence of lymphedema. The diagnostic performance using normative data-derived cut-offs (i.e., mean plus 0.5 standard deviation [SD] to mean plus $3 \mathrm{SD} ; \mathrm{n}=136,66 \%$ controls) and receiver operating characteristic (ROC) curve-derived cut-offs ( $\mathrm{n}=746,94 \%$ controls) was assessed. The impact of sex, age and BMI were investigated by comparing stratified and non-stratified normative data-derived cut-offs, and ROC curves generated from adjusted and unadjusted logistic regression models. Arm-to-leg ratios of ECW between mean plus $0.5 \mathrm{SD}$ and mean plus 1 SD showed fair to good sensitivity $(0.73-0.84)$ and poor to good specificity $(0.64$ to 0.84). Arm-to-leg ratios of ECW/ICW failed to detect lymphedema (sensitivity <0.5). Stratification by sex, or by sex and age, yielded similar results to non-stratified cut-offs. Cutoffs derived from adjusted ROC curves showed both good sensitivity (0.83-0.89) and specificity $(0.8-0.84)$.

CONCLUSION: These findings represent new BIS criteria for diagnosing lower-limb lymphedema that do not rely on comparison to baseline measures or the presence of a nonaffected, contralateral limb. 


\section{CONDENSED ABSTRACT:}

Unilateral lymphedema is commonly detected as an increase in extracellular water (ECW) relative to a comparable unaffected limb. This method is unsuitable for the detection of potential bilateral lymphedema, since both upper- or lower-limbs are affected. This study tested the diagnostic performance of comparing impedance to ECW in legs to that of ECW in arms, using bioimpedance spectroscopy, in the detection of bilateral lower-limbs lymphedema. Results demonstrated that prediction equations based on sex, age and body mass index-adjusted logistic regression models have excellent sensitivity and specificity, and are ready for use in the clinic and research settings. 


\section{INTRODUCTION}

Lymphedema is a condition of impaired lymph drainage, which initially presents as swelling, tightness, heaviness or throbbing in the affected areas ${ }^{1,2}$. Progressive changes can lead to deposition of adipose tissue and fibrosis, resulting in gross disfiguration and loss of function ${ }^{2}$. Lymphedema is estimated to affect up to 250 million people worldwide, with $90 \%$ of cases reported to affect the lower-limbs ${ }^{3}$. Approximately $1 \%$ of all cases are described as "primary lymphedema" and are idiopathic or heritable, while $99 \%$ of cases are described as "secondary lymphedema" and develop from acquired damage to or dysfunction of the lymphatic system. The most common causes of secondary lymphedema are parasitic infection (developing countries), cancer treatment and obesity (developed countries) ${ }^{3}$.

Accumulating evidence from research involving women with unilateral, upper-limb lymphedema after treatment for breast cancer suggests that early detection and intervention can reduce the risk of subclinical lymphedema progressing to a chronic advanced stage, which becomes particularly difficult and expensive to treat ${ }^{4-9}$. Currently in the clinic, lower-limb lymphedema is usually diagnosed after clinical examination following a complaint of swollen or heavy-feeling legs. In cases of unilateral lymphedema, the affected leg can be compared with the unaffected leg (e.g., using bioimpedance spectroscopy [BIS] or circumferences) to determine if the leg is swollen. However, bilateral lymphedema is more complicated to diagnose due to the lack of availability of a comparable limb.

Bioimpedance spectroscopy, which measures impedance of extracellular and intracellular water to an alternating electrical current passed through the body at a range of non-discernible frequencies, has previously been shown to be a non-invasive, portable technology capable of detecting subclinical lymphedema ${ }^{9}$. Subclinical lymphedema is considered present when the 
ratio of extracellular water $(\mathrm{ECW})$ between arms or between legs is outside 3 standard deviations (SDs) of the normative mean ${ }^{10,11}$. However, due to the unavailability of an unaffected contralateral limb for comparison, there is a notable lack of effective criteria for detecting bilateral lymphedema in the upper- or lower-limbs.

Cornish et al. ${ }^{12}$ proposed that since lymphedema is an accumulation of lymph in ECW with intracellular water (ICW) being unchanged, the ratio of ECW/ICW within the same limb could be used as an index of the presence of lymphedema and provided preliminary supporting evidence from a small number of subjects with unilateral breast cancer-related lymphedema. In a later study ${ }^{13}$, the method was extended to quantification of bilateral lymphedema although it was noted that ECW/ICW ratios varied with age, sex and limb dominance. Recently, Steele et al. ${ }^{14}$ published the first normative values for arm-to-leg ratios of ECW (dominant side $=$ $1.129 \pm 0.160$, non-dominant side $=1.165 \pm 0.174)$ and $\mathrm{ECW} / \mathrm{ICW}($ dominant side $=1.194 \pm$ 0.453 , non-dominant side $=1.117 \pm 0.367$ ) based on 808 self-reported healthy individuals with no history of lymphedema. Updated values for inter-arm $(\mathrm{ECW}=0.987 \pm 0.067, \mathrm{ECW} / \mathrm{ICW}=$ $0.957 \pm 0.188)$ and inter-leg $(\mathrm{ECW}=1.005 \pm 0.072, \mathrm{ECW} / \mathrm{ICW}=1.024 \pm 0.183)$ ratios, with and without stratification by sex, age and body mass index (BMI) were also reported. Key findings were that arm-to-leg ratios (but not inter-arm or inter-leg ratios) were significantly affected by sex and age in accord with the observations of Ward et al. ${ }^{10}$, and to a lesser extent by BMI, and that normalising ECW to ICW led to larger within and between-subject variation ${ }^{14}$. Based on these results, we predicted that arm-to-leg ratios of ECW and ECW/ICW, possibly stratified by sex and age, could be used to develop effective criteria for identifying bilateral lymphedema. 
Therefore, the first aim of this case-control study was to assess whether arm-to-leg ratios of ECW and ECW/ICW differed between cases and controls. The second aim was to test whether binary cut-offs of arm-to-leg ratios (ECW and ECW/ICW) derived using two approaches (i.e., the normal distribution method and the receiver operating characteristic (ROC) curve method) can accurately detect clinically diagnosed lower-limb lymphedema. The third aim was to investigate whether stratifying normative data-derived cut-offs by sex and age, or adjusting ROC curve-derived cut-offs by sex, age and BMI, improves the diagnostic performance of cutoffs.

\section{METHODS}

\section{Participants}

We conducted a dual approach, case-control study consisting of cases of bilateral, lower-limb lymphedema and healthy controls who self-reported absence of lymphedema (Figure 1). Cases $(n=46)$ consisted of a convenience sample of males and females with clinically diagnosed allcause, bilateral, lower-limb lymphedema. Controls consisted of a normative, pooled dataset that has been described previously ${ }^{14}$. Briefly, a sample $(n=898)$ of self-reported healthy males and females with no history of lymphedema, removal or irradiation of lymph nodes or cancer, were recruited from five centres across Australia and New Zealand. Our first approach involved randomising controls 9:1 into a 'training' set $(n=808)$, which was previously used for calculating normative means and $\mathrm{SDs}^{14}$, and a 'test' set $(\mathrm{n}=90)$, which was retained for use in testing the prediction of normative data-derived cut-offs in the present study in an independent set of observations. A 9:1 split was chosen to maximise the number of controls used for generating normative data-derived cut-offs and to allow stratification of cut-offs by sex, age and BMI. Further, a test set of 90 controls was considered sufficient, given we only had access 
to 46 cases of bilateral lower-limb lymphedema. Our second approach involved using all controls with complete data $(n=700$; data for 198 participants were excluded due to missing sex, age, BMI or specific BIS measurements; see Figure 1) and all cases $(n=46)$. Appropriate ethical approvals were obtained from the relevant institutions for the conduct of all initial studies, as well as the present study (ACTRN1500001072), and all participants provided written informed consent.

\section{Procedures}

All contributing studies implemented the same validated protocol as published previously ${ }^{14}$ and as outlined by Impedimed ${ }^{15}$ to measure impedance of total body water (TBW) $\left(\mathrm{R}_{\text {inf }}\right)$, ICW $\left(\mathrm{R}_{\mathrm{i}}\right)$ and ECW $\left(\mathrm{R}_{0}\right)$ in arms and legs using either a portable, Impedimed SFB7, SFB3 or U400 BIS device. BIS measurements were taken with the participant lying supine with legs apart. For any given measure, two measurement electrodes were placed at either end of the arm or leg (i.e., wrist for arm measures and ankle for leg measures) with a current drive electrode placed at the base of the middle finger or toe, respectively, and another placed on the ipsilateral or contralateral foot or hand, respectively ${ }^{16}$. Each limb segment was measured in this manner and the resistances corresponding to ECW $\left(\mathrm{R}_{0}\right)$ and to TBW ( $\left.\mathrm{R}_{\text {inf }}\right)$ were determined. ICW resistance $\left(\mathrm{R}_{\mathrm{i}}\right)$ was subsequently calculated with the manufacturer's software (Bioimp 4.15.0.0, ImpediMed Ltd., Brisbane), which uses the formula: $R_{i}=\left(R_{0} x R_{i n f}\right) /\left(R_{0}-R_{\text {inf }}\right)$. Sex, age, height, weight and self-reported dominant hand were recorded for each participant.

\section{Data Analysis}

Characteristics of cases and controls were summarised and compared using unpaired t-tests and chi-squared tests. The following inter-limb ratios were calculated for each participant: dominant arm/non-dominant arm $\left(\mathrm{R}_{0}\right.$ and $\left.\mathrm{R}_{\mathrm{i}}: \mathrm{R}_{0}\right)$; dominant leg/non-dominant leg $\left(\mathrm{R}_{0}\right.$ and 
$\left.\mathrm{R}_{\mathrm{i}}: \mathrm{R}_{0}\right)$; dominant arm/dominant leg $\left(\mathrm{R}_{0}\right.$ and $\left.\mathrm{R}_{\mathrm{i}}: \mathrm{R}_{0}\right)$; and non-dominant arm/non-dominant leg $\left(\mathrm{R}_{0}\right.$ and $\left.\mathrm{R}_{\mathrm{i}}: \mathrm{R}_{0}\right)$. Inter-limb ratios of cases were compared with controls using boxplots and Wilcoxon signed-rank tests.

\section{Normative data-derived cut-offs}

Binary cut-offs ranging from mean plus 0.5 to $3 \mathrm{SD}$ were calculated based on the stratified (sex, and sex and age) and non-stratified means and SDs of the normative inter-limb ratios published previously ${ }^{14}$. All cut-offs were evaluated for their ability to distinguish cases of bilateral lowerlimb lymphedema $(n=46)$ from controls in the test data set $(n=90)$ by calculating and comparing sensitivity and specificity (total $\mathrm{n}=136)$.

\section{ROC curve-derived cut-offs}

All participants with complete demographic (sex, age and BMI) and bioimpedance data, that is controls from training and test data sets $(n=700)$ plus cases $(n=46)$, were used for generating ROC curve-derived cut-offs (total $\mathrm{n}=746$ ). Univariable (dependent variable $=$ lymphedema status; independent variable $=$ inter-limb $\mathrm{R}_{0}$ or $\mathrm{R}_{\mathrm{i}}: \mathrm{R}_{0}$ ratio) and multivariable (dependent variable $=$ lymphedema status; independent variables $=$ inter-limb $\mathrm{R}_{0}$ or $\mathrm{R}_{\mathrm{i}}: \mathrm{R}_{0}$ ratio, sex, age and BMI) logistic regression models were fit to establish factors associated with lymphedema status and produce probabilities for generating ROC curves. McFadden's pseudo $\mathrm{R}^{2}$ values were calculated to assess model fit. Pseudo $\mathrm{R}^{2}$ values for logistic regression models are typically lower than traditional $\mathrm{R}^{2}$ values for linear regression models. According to McFadden ${ }^{17}$, pseudo $\mathrm{R}^{2}$ values between 0.2 and 0.4 represent an excellent fit. All covariates were retained in the multivariable logistic regression models (independent of their association with lymphedema status) based on evidence that sex, age and BMI significantly affect arm-toleg $\operatorname{ratios}^{14}$. ROC curves were created using raw impedance ratios (equivalent to the unadjusted 
logistic regression models) and probabilities generated from the multivariable logistic regression models. ROC curves were compared using the bootstrap test for two correlated ROC curves and the area under the curve (AUC) was calculated as a measure of diagnostic performance for all predictors $($ AUC of $0.5-0.6=$ fail, $0.6-0.7=$ poor, $0.7-0.8=$ fair, $0.8-0.9=$ good, $0.9-1=$ excellent). Optimal cut-offs were selected using clinical reasoning, where we considered sensitivity to be more important than specificity in the context of diagnosing lymphedema. A priori we considered sensitivity above $80 \%$ and specificity above $70 \%$ as being indicative of a clinically useful diagnostic threshold for lymphedema. All analyses were conducted in $\mathrm{R}$ version 3.3.1. The R packages, $\mathrm{pROC}$ and epiR, were used to construct ROC curves and calculate measures of diagnostic performance ${ }^{18}$.

\section{RESULTS}

\section{Characteristics of participants}

Data from a total of 944 participants (controls $=898$, cases $=46$ ) were assessed in the current study. The characteristics of all participants are presented in Table 1. There was a greater proportion of females among cases compared with controls ( $89 \%$ versus $65 \%, p=0.002)$, cases were significantly older $(64 \pm 13$ versus $48 \pm 15$ years; $p<0.001)$ and had higher BMI $(34 \pm 8$ versus $\left.26 \pm 4 \mathrm{~kg} / \mathrm{m}^{2} ; \mathrm{p}<0.001\right)$. Comparable differences in demographic variables between cases and controls were observed when assessed in each of these subsets (i.e., between the cases and controls used in the normal distribution approach and the ROC curve approach).

\section{Comparison of inter-limb ratios between cases and controls}

Inter-limb ratios of cases $(n=46)$ versus controls with complete data $(n=700)$ are presented in Figure 2. Arm-to-leg ratios of $\mathrm{R}_{0}$ were larger for cases compared with controls, with a mean difference of $0.318(p<0.01)$ on the dominant side of the body and $0.336(p<0.01)$ on the non- 
dominant side. Arm-to-leg ratios of $\mathrm{R}_{\mathrm{i}}: \mathrm{R}_{0}$ also showed differences between cases and controls, with mean differences of $0.470(\mathrm{p}<0.01)$ and $0.548(\mathrm{p}<0.01)$ on the dominant and nondominant sides, respectively. For completeness, inter-arm and inter-leg ratios were also compared (data not shown). Inter-arm ratios of $\mathrm{R}_{0}$, inter-leg ratios of $\mathrm{R}_{0}$, and inter-arm ratios of $\mathrm{R}_{\mathrm{i}}: \mathrm{R}_{0}$ did not differ between cases and controls, while inter-leg ratios of $\mathrm{R}_{\mathrm{i}}: \mathrm{R}_{0}$ differed by $0.158(\mathrm{p}<0.01)$

\section{Normative data-derived cut-offs}

Sensitivity and specificity were calculated to describe the ability of cut-offs derived from normative inter-leg $\mathrm{R}_{0}$ ratios, arm-to-leg $\mathrm{R}_{0}$ ratios and arm-to-leg $\mathrm{R}_{\mathrm{i}}: \mathrm{R}_{0}$ ratios (see Supplementary Table 1 for sensitivity and specificity findings). Both the inter-leg $\mathrm{R}_{0}$ ratios and the arm-to-leg $\mathrm{R}_{\mathrm{i}}: \mathrm{R}_{0}$ ratios performed poorly with very low sensitivity $(<0.5)$ for all cut-offs. Arm-to-leg ratios of $\mathrm{R}_{0}$ performed relatively well at cut-offs between mean plus $0.5 \mathrm{SD}$ and mean plus $1 \mathrm{SD}$, with sensitivity ranging from 0.73 to 0.84 and specificity ranging from 0.64 to 0.84 . Cut-offs stratified by demographic factors (that is, sex, and sex and age) also performed relatively well, with sensitivity ranging from 0.65 to 0.80 and 0.65 to 0.75 between mean plus 0.5 to mean plus 1 SD for sex stratified, and sex and age stratified cut-offs, respectively. Specificity ranged from 0.61 to 0.81 and 0.66 to 0.80 , between mean plus 0.5 and mean plus 1 SD for sex stratified, and sex and age stratified cut-offs, respectively. Therefore, stratification by sex, or by sex and age, did not appear to improve diagnostic performance of normative dataderived cut-offs.

\section{ROC curve-derived cut-offs}

Table 2 presents model coefficients for the four adjusted logistic regression models (dependent variable $=$ lymphedema status, independent variables $=$ inter-limb ratios, sex, age and BMI), 
alongside McFadden's pseudo $\mathrm{R}^{2}$ values for the adjusted and unadjusted models. With the exception of one (unadjusted model for dominant $\mathrm{R}_{\mathrm{i}}: \mathrm{R}_{0}$ ratio), all pseudo $\mathrm{R}^{2}$ values were above 0.2 , indicating excellent fit. However, all pseudo $\mathrm{R}^{2}$ values for the adjusted models were approximately twice that of the unadjusted models, indicating superiority of the adjusted models (Table 2). Unadjusted and adjusted ROC curves are presented in Figure 3 and the diagnostic performance of predictors is summarised in Table 3. All AUC values were significantly larger than $0.5(\mathrm{p}<0.001)$, and the adjusted models performed significantly better than unadjusted ratios at classifying cases of lymphedema $(\mathrm{p}<0.001)$. There were no significant differences between comparable ROC curves for $\mathrm{R}_{0}$ and $\mathrm{R}_{\mathrm{i}}: \mathrm{R}_{0}(\mathrm{p}>0.05)$. Cut-offs with maximal sensitivity and adequate specificity were derived from ROC curves and are presented in Table 4. Cut-offs derived from unadjusted ROC curves showed fair to good sensitivity $(0.72-0.80)$ and poor to fair specificity $(0.63-0.73)$, similar to that of the normative data-derived cut-offs. Cut-offs derived from adjusted ROC curves showed both good sensitivity (0.83-0.89) and specificity $(0.8-0.84)$.

\section{DISCUSSION}

Detection of bilateral lymphedema is complicated by the unavailability of an unaffected comparable limb. The current study investigated whether arm-to-leg ratios of ECW or ECW/ICW can be used to detect lymphedema in individuals at risk of bilateral lymphedema. Ratios differed significantly between individuals with and without bilateral lower-limb lymphedema, providing support for the use of arm-to-leg ratios. Diagnostic cut-off values were derived using two different approaches. The first approach used binary cut-offs derived from normative data using the normal (Gaussian) distribution method. This involved calculation of non-stratified, sex-stratified, and sex and age-stratified cut-offs ranging from the normative mean plus $0.5 \mathrm{SD}$ up to plus $3 \mathrm{SD}$. Arm-to-leg ratios of $\mathrm{R}_{0}$ performed relatively well at cut- 
offs between mean plus $0.5 \mathrm{SD}$ to mean plus $1 \mathrm{SD}$, which equates to cut-off values of 1.209 to 1.289 on the dominant side of the body and 1.252 to 1.339 on the non-dominant side. Neither stratification by demographic variables nor normalisation of ECW to ICW improved diagnostic performance of arm-to-leg ratios.

The second approach for deriving cut-offs involved construction of ROC curves using unadjusted and adjusted arm-to-leg ratios and selection of optimal cut-offs based on clinicallyinformed decision making. Comparison of McFadden's $\mathrm{R}^{2}$ values (as a measure of model fit) and AUCs (as a measure of accuracy) of unadjusted and adjusted logistic regression models suggests that adding sex, age and BMI to the model significantly improves diagnostic performance of arm-to-leg ratios. Indeed, unadjusted ratios produced AUCs of 0.79 to 0.86 , indicative of fair to good accuracy, while adjusted models produced AUCs between 0.89 and 0.93, indicative of good to excellent accuracy. In selecting optimal cut-offs, we considered the relative consequences of false-negative and false-positive test results (e.g., missed opportunity for prevention and development of chronic lymphedema versus unnecessary follow-up screening and/or provision of a non-invasive therapy such as lymphatic drainage massage) and chose to maximise sensitivity while maintaining acceptable specificity $(>0.7)$. Interestingly, our ROC curve-derived cut-offs for unadjusted $\mathrm{R}_{0}$ ratios $($ dominant $=1.217$, non-dominant $=$ 1.211) were similar to cut-offs based on the normative mean plus $0.5 \mathrm{SD}$ (dominant $=1.209$, non-dominant $=1.252)$. In contrast, the ROC curve-derived cut-offs for $\mathrm{R}_{\mathrm{i}}: \mathrm{R}_{0}$ ratios (dominant $=0.903$, non-dominant $=0.905)$ were smaller than normative data-derived cut-offs $($ dominant $=1.421$, non-dominant $=1.301)$, and significantly better at detecting lymphedema. Cut-offs derived from adjusted ROC curves showed significantly better sensitivity and specificity than cut-offs derived from unadjusted ROC curves and normative data-derived cut-offs. Therefore, we propose that the following predictive equations based on regression coefficients from the 
adjusted logistic regression models, could be used for accurately detecting bilateral, lower-limb lymphedema:

Dominant side of the body:

predictor $=-18.41+5.58\left(\operatorname{arm~} R_{0} / \operatorname{leg} R_{0}\right)-0.45($ male $=1 \mid$ female $=0)+0.03($ age $)+0.24(B M I)$

probability $=\exp ($ predictor $) /(1+\exp ($ predictor $))$

probability $>\mathbf{0 . 0 4 5}=$ lymphedema

Non-dominant side of the body:

predictor $=-18.59+6.11\left(\right.$ arm $\left.R_{0} / \operatorname{leg} R_{0}\right)-0.44($ male $=1 \mid$ female $=0)+0.02($ age $)+0.24(B M I)$

probability $=\exp ($ predictor $) /(1+\exp ($ predictor $))$

probability $>\mathbf{0 . 0 4 2}=$ lymphedema

Furthermore, despite having reduced sensitivity and specificity, the following raw arm-to-leg ratios of ECW could be used for detection of lower-lymphedema if demographic data were unavailable or as a quick and easy, but less reliable preliminary diagnostic tool.

Dominant side of the body:

$\operatorname{arm} \mathrm{R}_{0} / \operatorname{leg} \mathrm{R}_{0}>\mathbf{1 . 2 1 7}=$ lymphedema

Non-dominant side of the body:

$\operatorname{arm} \mathrm{R}_{0} / \operatorname{leg} \mathrm{R}_{0}>\mathbf{1 . 2 1 1}=$ lymphedema

While a limited number of studies have explored the potential of using BIS for detecting lowerlimb lymphedema ${ }^{10,12,13,19,20}$, we present the first sensitive and specific BIS-based diagnostic cut-offs, which do not rely on baseline (pre-lymphedema) measures and can be easily applied in the clinic. Novakova et al. ${ }^{20}$ have previously tested two BIS-based methods of detecting 
lower-limb lymphedema in 29 patients who were assessed using BIS prior to and 6 months after surgery for vulvar cancer. The first method diagnosed lymphedema if leg ECW at 6months post-surgery was greater than $1.645 \mathrm{SD}$ from the leg ECW pre-surgery, while the second method diagnosed lymphedema if the leg ECW at 6 months was $140 \%$ of leg ECW presurgery. Both methods showed a sensitivity of 0.67 , but the second method showed greater specificity (0.6 versus 0.45$)$. However, not only does this method rely on the collection of a baseline measurement, it appears to be less sensitive and less specific than the criteria presented herein.

BIS-based diagnosis has been used extensively in the detection of breast cancer-related lymphedema. The diagnostic performance of our proposed criteria for detecting lower-limb lymphedema (sensitivity $=0.87-89$; specificity $=0.83 ; \mathrm{AUC}=0.93$ ) is in line with the published performance of cut-offs for unilateral, upper-limb lymphedema. For example, Smoot et al. ${ }^{21}$ assessed the diagnostic ability of the BIS cut-offs for unilateral, upper-limb lymphedema published by Cornish et al. ${ }^{22}$, and found sensitivity of 0.31 to 0.87 , specificity of 0.47 to 1 , and an AUC of 0.88. More recently, Dylke et al. ${ }^{23}$ assessed the ability of a range of BIS cut-offs to detect unilateral, upper-limb lymphedema and found that sensitivity ranged from 0.93 to 0.96 and specificity ranged from 0.67 to 0.87 .

While the findings presented here are relevant and ready for use in the research and clinical setting, limitations to this work need to be acknowledged. First, our control and case data come from convenience sampling with males under-represented in each dataset, and cases having higher BMI on average compared with controls. Group differences between cases and controls may partly explain why the prediction equation performs better than the unstratified and age and sex stratified diagnostic thresholds, as presented here. Second, while the majority of our lymphoedema cases were more likely to have stage II+ lymphedema, we have limited 
information on the cause or composition of their lymphedema. Consequently, we were unable to explore any potential impact of these factors on the performance of the diagnostic criteria. Future research in evaluating the diagnostic potential of the proposed cut-offs in a sample with mixed lymphoedema stage, as well as testing the performance of the cut-offs in the bilateral, upper-limb lymphedema setting, is clearly warranted.

It is well known that the outcomes of sensitivity and specificity analysis are highly dependent upon the choice of the reference method ${ }^{24}$. While there exists no gold standard for the diagnosis of lymphoedema, a strength of this work is that our cases had clinically confirmed lower limb lymphoedema, as assessed by an experienced lymphoedema therapist. As noted earlier, we prioritised sensitivity over specificity in identifying the proposed diagnostic thresholds. This is standard for analysis that seeks to identify a diagnostic threshold, but was also particularly appropriate here, given our controls did not undergo a clinical evaluation and may have included individuals with increases in extracellular fluid unrelated to cancer. Nonetheless, by prioritising sensitivity over specificity, and in acknowledgement that BIS measures fluid changes and differences, irrespective of cause, the diagnostic threshold specified may be more likely to over- than under-diagnose cases. Overdiagnosis through use of these criteria could be reduced by also considering patient-reported symptoms and/or other clinical assessments. Further, we have reported the sensitivity and specificity of a range of cutoffs in the supplementary table to allow for future use of different cut-offs based on different criteria or priorities.

In conclusion, we present BIS criteria for detecting lymphedema in individuals at risk of lowerlimb lymphedema that do not rely on comparison to baseline or contralateral limb measures. Further, findings suggest that unstratified arm-to-leg ratios of ECW are sensitive and specific, 
while the prediction equations based on sex, age and BMI-adjusted logistic regression models showed excellent sensitivity and specificity, and are easily translatable to the clinic.

\section{ACKNOWLEDGEMENTS:}

We wish to thank and acknowledge the following organisations for contribution of funds that enabled this work to be completed: Impedimed Limited (funds supported the data analysis for this body of work but not the original data collection studies); Cancer Council Queensland (SH fellowship and RB grant), National Health and Medical Research Council (MJ fellowship), Department of Health and Aging (RB grant) and Fonterra Co-operative Group and the New Zealand Ministry for Primary Industries (funds supported the New Zealand Primary Growth Partnership post-farm gate dairy programme). Funding received had no influence over data analysis and reporting.

\section{AUTHOR DISCLOSURE STATEMENT:}

No competing financial interests exist. LW provides consultancy services to ImpediMed Limited. Impedimed Limited donated dual-tab electrodes to SG. SDP holds the Fonterra Chair in Human Nutrition at the University of Auckland. AR is an employee of Fonterra CoOperative Group. AO is the founder and managing director of SurgicalPerformance Pty Ltd., an Australian, private company that provides surgeons with a platform for collection of surgical outcome audit data; AO received travel grants from the O.R. Company (formerly Tyco Healthcare) and is a consultant for Covidien, NSW, Australia. 


\section{REFERENCES}

1. Ly CL., Kataru RP., Mehrara BJ., Inflammatory Manifestations of Lymphedema. Int J Mol Sci, 2017; 18(1).

2. Warren, AG., et al., Lymphedema: a comprehensive review. Ann Plast Surg, 2007; 59(4): 464-72.

3. Greene AK., Slavin SA., Brorson H., Lymphedema: Presentation, Diagnosis, And Treatment. 2015: Springer.

4. Dönmez AA., Kapucu S., The effectiveness of a clinical and home-based physical activity program and simple lymphatic drainage in the prevention of breast cancerrelated lymphedema: A prospective randomized controlled study. European Journal of Oncology Nursing, 2017; 31: 12-21.

5. Stout Gergich NL., et al., Preoperative assessment enables the early diagnosis and successful treatment of lymphedema. Cancer, 2008; 112(12): 2809-19.

6. Kaufman DI., et al., Utilization of bioimpedance spectroscopy in the prevention of chronic breast cancer-related lymphedema. Breast Cancer Research and Treatment, 2017; 166(3): 809-815.

7. Rafn BS., et al., Prospective surveillance and targeted physiotherapy for arm morbidity after breast cancer surgery: a pilot randomized controlled trial. Clinical Rehabilitation, 2018; 32(6): 811-826.

8. Whitworth PW., Cooper A, Reducing chronic breast cancer-related lymphedema utilizing a program of prospective surveillance with bioimpedance spectroscopy. Breast J, 2018; 24(1): 62-65.

9. Ward LC., Early diagnosis in the latent phase, in Lymphedema: a concise compendium of theory and practice. In: Lee BB., Bergan J., Rockson S. eds. SpringerVerlag GmbH: Vienna; 2018. 
10. Ward LC., et al., Reference ranges for assessment of unilateral lymphedema in legs by bioelectrical impedance spectroscopy. Lymphat Res Biol, 2011; 9(1): 43-6.

11. Ward LC., et al., Confirmation of the reference impedance ratios used for assessment of breast cancer-related lymphedema by bioelectrical impedance spectroscopy. Lymphat Res Biol, 2011; 9(1): 47-51.

12. Cornish BH., et al., A new technique for the quantification of peripheral edema with application in both unilateral and bilateral cases. Angiology, 2002; 53(1): 41-7.

13. Ward LC., et al., Assessment of bilateral limb lymphedema by bioelectrical impedance spectroscopy. Int J Gynecol Cancer, 2011; 21(2): 409-18.

14. Steele ML., et al., Normative Interlimb Impedance Ratios: Implications for Early Diagnosis of Uni- and Bilateral, Upper and Lower Limb Lymphedema. Lymphat Res Biol, 2018; https://doi.org/10.108g//rb.2017.0082

15. Impedimed Ltd. Impedimed. 2017 [17th October 2017]; Available from: https://www.impedimed.com/.

16. Cornish BH., et al., Optimizing electrode sites for segmental bioimpedance measurements. Physiological measurement, 1999; 20(3): 241-50.

17. McFadden D., Quantitative methods for analysing travel behaviour of individuals. In: Hensher D., Stopher P, eds. Behavioural Travel Modelling; Croom Helm Ltd: London; 1977.

18. Robin X., et al., pROC: an open-source package for R and S+ to analyze and compare ROC curves. BMC Bioinformatics, 2011; 12: 77.

19. Suehiro K., et al., Application of the L-Dex Score for the Assessment of Bilateral Leg Edema. Lymphat Res Biol, 2018; 16(1): 65-68. 
20. Novackova M., et al., A prospective study in detection of lower-limb lymphedema and evaluation of quality of life after vulvar cancer surgery. Int J Gynecol Cancer, 2012; 22(6): 1081-8.

21. Smoot BJ., Wong JF., Dodd MJ., Comparison of diagnostic accuracy of clinical measures of breast cancer-related lymphedema: area under the curve. Arch Phys Med Rehabil, 2011; 92(4): 603-10.

22. Cornish BH., et al., Early diagnosis of lymphedema using multiple frequency bioimpedance. Lymphology, 2001; 34(1): 2-11.

23. Dylke ES., et al., Diagnosis of upper limb lymphedema: development of an evidencebased approach. Acta Oncol, 2016; 55(12): 1477-1483.

24. van Stralen KJ., et al., Diagnostic methods I: Sensitivity, specificity, and other measures of accurary. Kidney Int, 2009; 75: 1257-1263. 


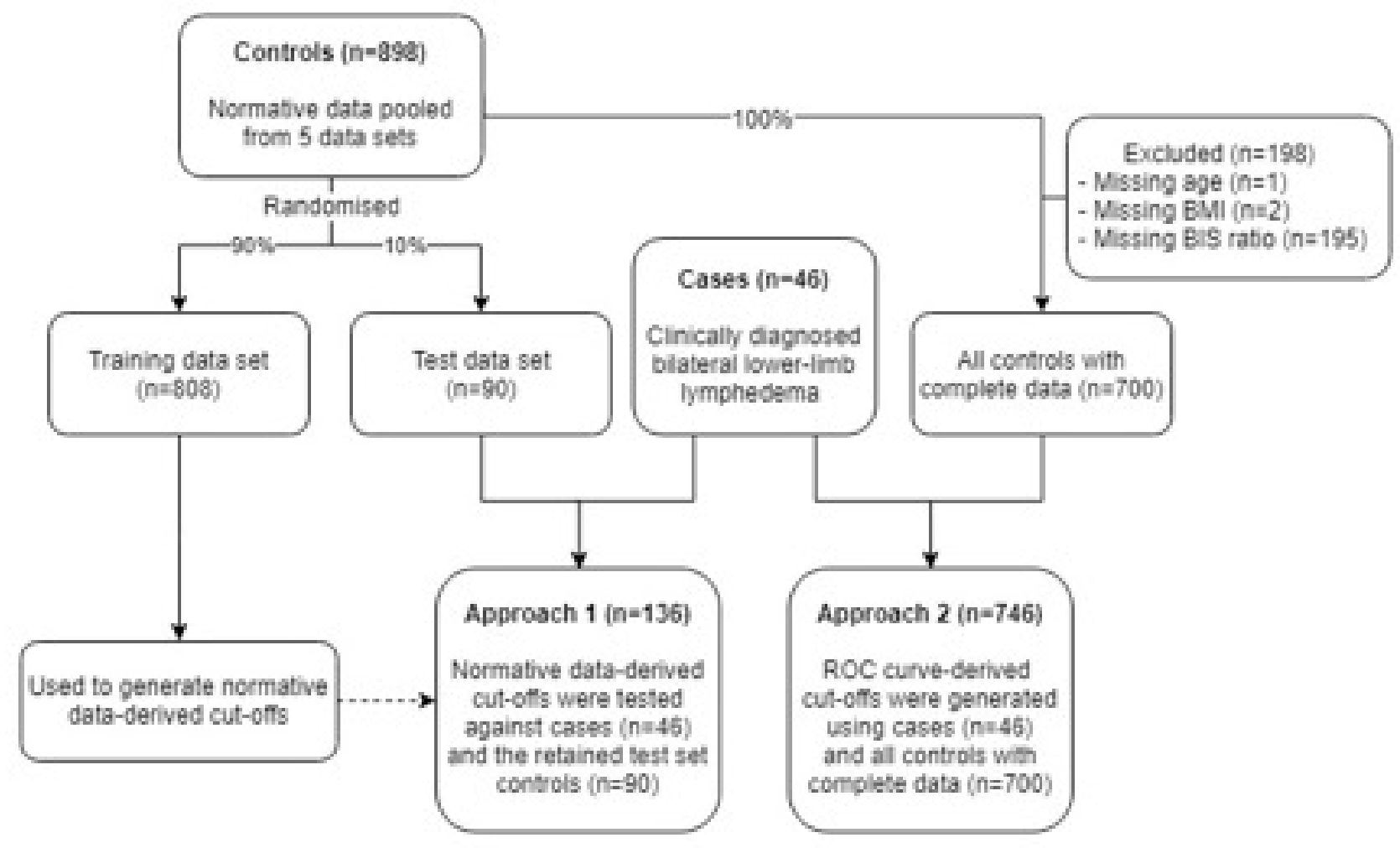

FIG. 1. Flowchart showing the flow of participant data through the study. BIS, bioimpedance spectroscopy; BMI, body mass index; ROC, receiver operating characteristic. 

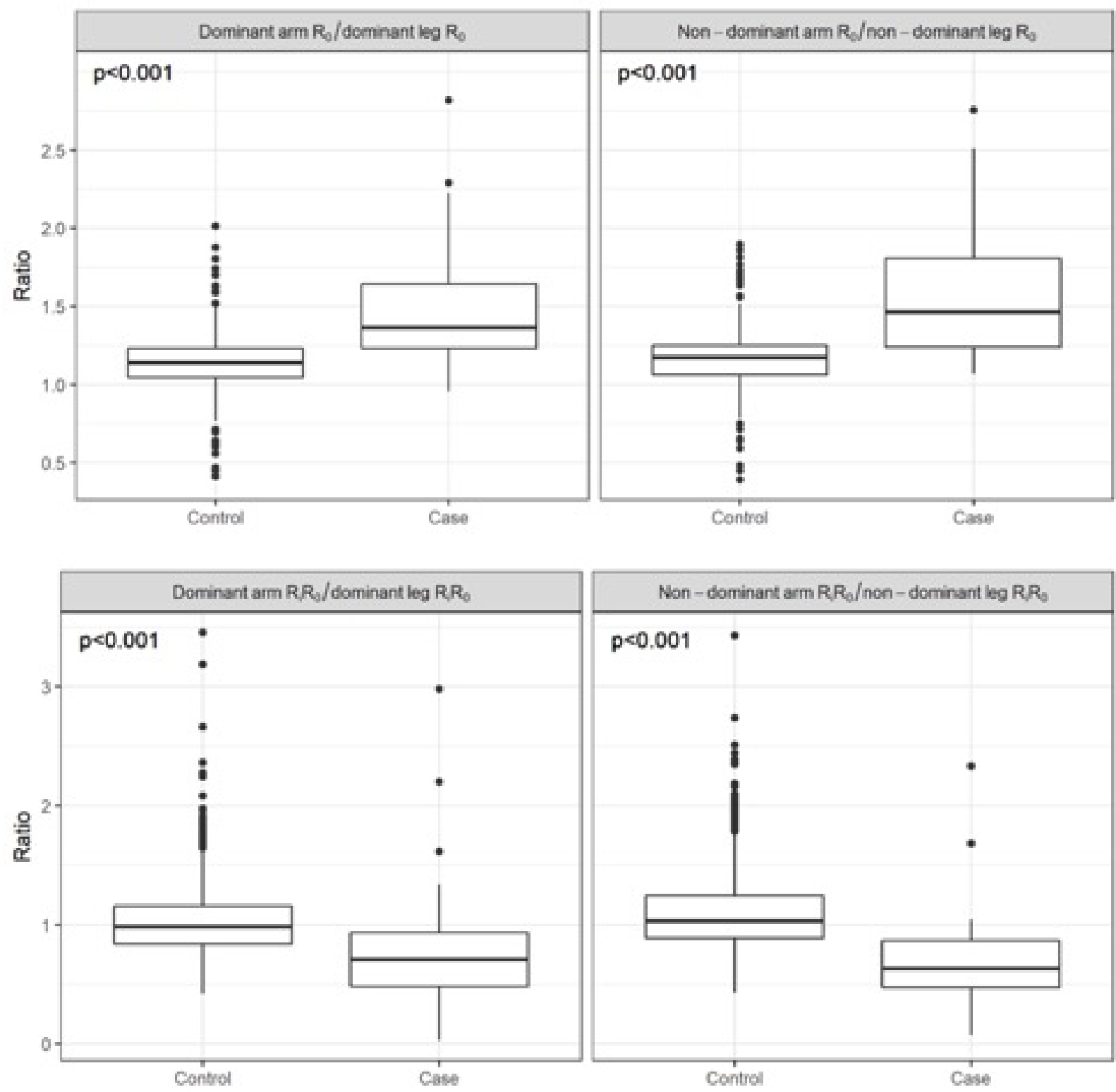

FIG. 2. Interlimb ratios of R0 and Ri:R0 of controls compared with lymphedema cases. The Wilcoxon signed-rank test was used to test whether inter-limb ratios of lymphedema cases were statistically different from controls $(\mathrm{p}<0.05)$. 

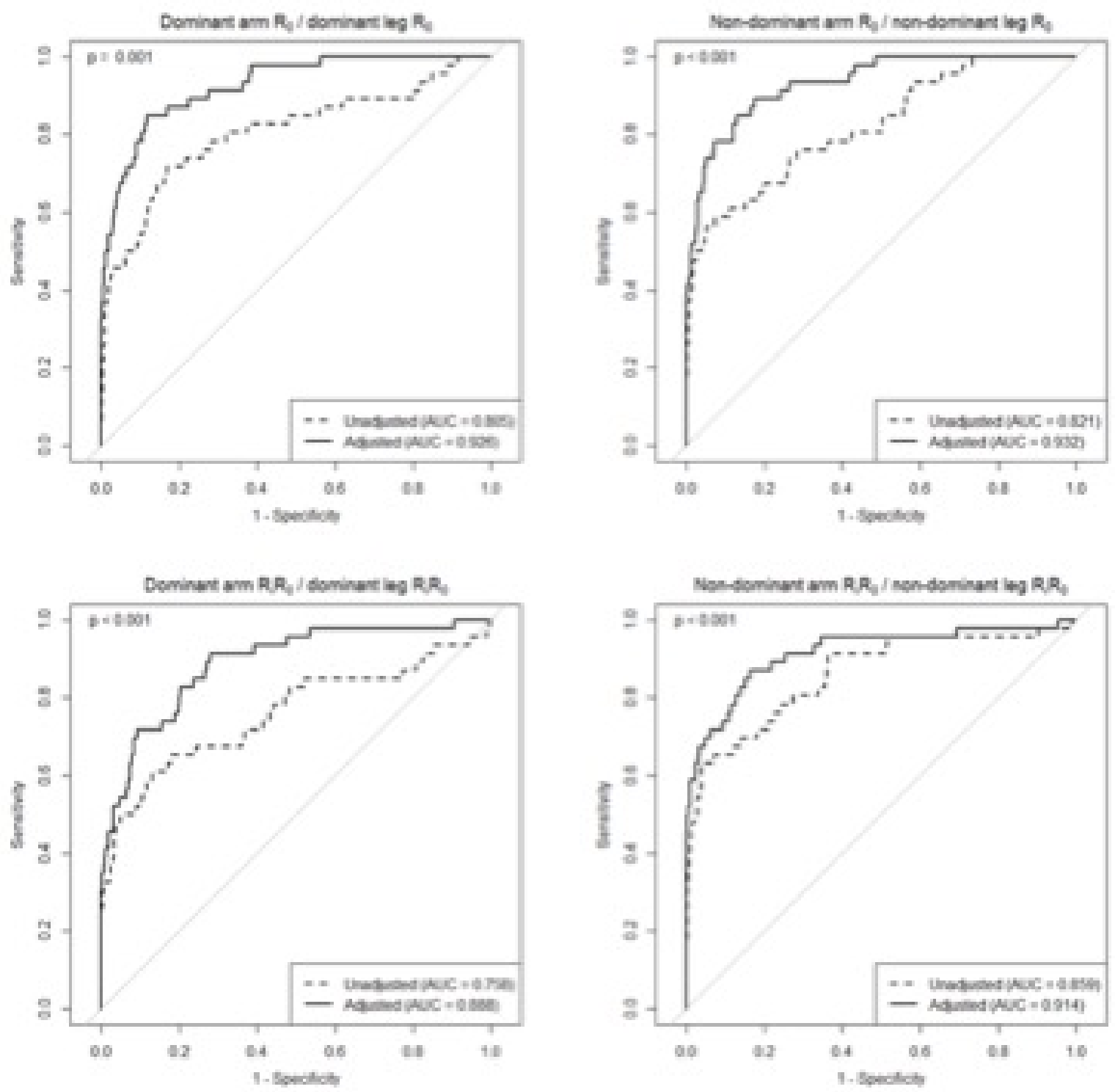

FIG. 3. ROC curves for inter-limb ratios of R0 and Ri:R0. Unadjusted and adjusted ROC

curves were compared by using the bootstrap test for two correlated ROC curves. AUC, area under the ROC curve. 
Table 1. Characteristics of participants.

\begin{tabular}{lcccc}
\hline Characteristic & All & Controls & Cases & p-value* \\
& $(\mathrm{n}=944)$ & $(\mathrm{n}=898)$ & $(\mathrm{n}=46)$ & \\
\hline Sex & $658(66)$ & $587(65)$ & $41(89)$ & 0.002 \\
Female, $\mathrm{n}(\%)$ & & & \\
Age (years) & $48 \pm 15,18-87$ & $48 \pm 15,18-87$ & $64 \pm 13,26-84$ & $<0.001$ \\
Mean \pm SD, Range & & & & \\
Body mass index $\left(\mathrm{kg} / \mathrm{m}^{2}\right)$ & & & & \\
Mean \pm SD, Range & $26 \pm 5,16-51$ & $26 \pm 4,16-46$ & $34 \pm 8,20-51$ & \\
Dominant hand & & & & \\
Right, $\mathrm{n}(\%)$ & $884(93)$ & $843(94)$ & & \\
\hline
\end{tabular}

*Two-sample t-tests (continuous variables) and chi-squared tests (categorical variables) were used to compare characteristics of cases and controls. 
Table 2. Model coefficients and fit.

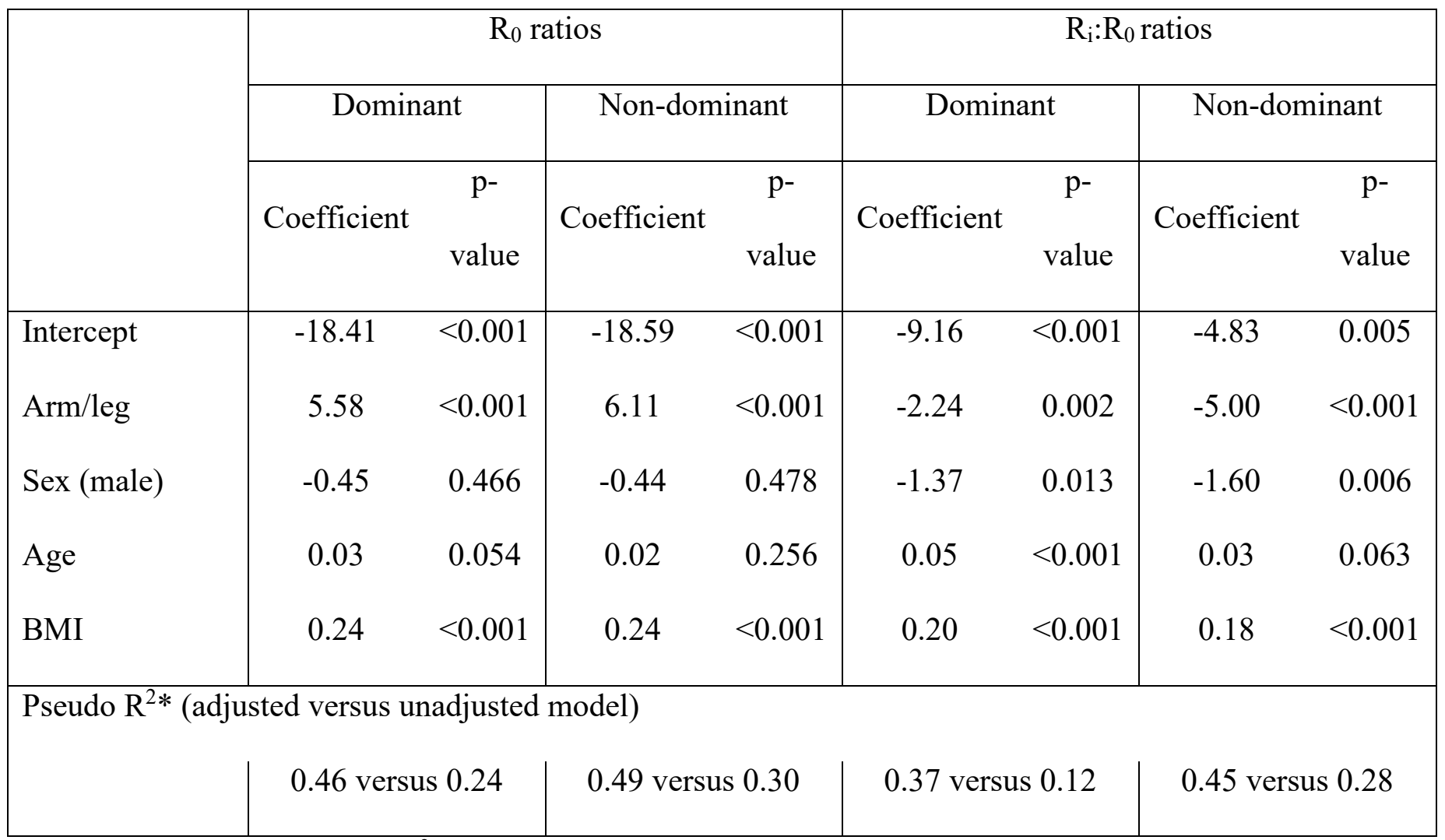

*McFadden's pseudo $\mathrm{R}^{2}$ values between 0.2 and 0.4 represent an excellent fit. 
Table 3. Diagnostic performance of unadjusted and adjusted inter-limb ratios of $R_{0}$ and $\mathrm{Ri}: \mathrm{R} 0$.

\begin{tabular}{|c|c|c|c|}
\hline Predictor & $\operatorname{AUC}(95 \% \mathrm{CI})$ & $\mathrm{SE}$ & p-value \\
\hline \multicolumn{4}{|l|}{ Unadjusted ratios } \\
\hline Dominant arm $\mathrm{R}_{0} /$ dominant leg $\mathrm{R}_{0}$ & $0.81(0.73,0.89)$ & 0.041 & $<0.001$ \\
\hline Non-dominant arm $\mathrm{R}_{0} /$ non-dominant leg $\mathrm{R}_{0}$ & $0.82(0.75,0.89)$ & 0.035 & $<0.001$ \\
\hline Dominant arm $\mathrm{R}_{\mathrm{i}}: \mathrm{R}_{0} /$ dominant leg $\mathrm{R}_{\mathrm{i}}: \mathrm{R}_{0}$ & $0.79(0.67,0.85)$ & 0.047 & $<0.001$ \\
\hline Non-dominant arm $\mathrm{R}_{\mathrm{i}}: \mathrm{R}_{0} /$ non-dominant leg $\mathrm{R}_{\mathrm{i}}: \mathrm{R}_{0}$ & $0.86(0.79,0.93)$ & 0.034 & $<0.001$ \\
\hline \multicolumn{4}{|l|}{ Model adjusted for sex, age and body mass index } \\
\hline Dominant arm $\mathrm{R}_{0} /$ dominant leg $\mathrm{R}_{0}$ & $0.93(0.89,0.96)$ & 0.019 & $<0.001$ \\
\hline Non-dominant arm $\mathrm{R}_{0} /$ non-dominant leg $\mathrm{R}_{0}$ & $0.93(0.90,0.97)$ & 0.018 & $<0.001$ \\
\hline Dominant arm $\mathrm{R}_{\mathrm{i}}: \mathrm{R}_{0} /$ dominant leg $\mathrm{R}_{\mathrm{i}}: \mathrm{R}_{0}$ & $0.89(0.84,0.94)$ & 0.027 & $<0.001$ \\
\hline Non-dominant arm $\mathrm{R}_{\mathrm{i}}: \mathrm{R}_{0} /$ non-dominant leg $\mathrm{R}_{\mathrm{i}}: \mathrm{R}_{0}$ & $0.91(0.86,0.97)$ & 0.027 & $<0.001$ \\
\hline
\end{tabular}

$\overline{\mathrm{A} p \text {-value }<0.05 \text { indicates that the true } \mathrm{AUC} \text { is not equal to } 0.5 ; \mathrm{AUC}=\text { area under the receiver }}$ operating characteristic curve; $\mathrm{CI}=$ confidence interval; $\mathrm{SE}=$ standard error. 
Table 4. Sensitivity and specificity of receiver operating characteristic curve-derived cut-offs.

\begin{tabular}{|c|c|c|c|}
\hline Predictor & Cut-off & $\begin{array}{l}\text { Sensitivity } \\
(95 \% \mathrm{CI})\end{array}$ & $\begin{array}{l}\text { Specificity } \\
(95 \% \mathrm{CI})\end{array}$ \\
\hline \multicolumn{4}{|l|}{ Unadjusted ratios } \\
\hline Dominant arm $\mathrm{R}_{0}$ / dominant leg $\mathrm{R}_{0}$ & 1.217 & $\begin{array}{c}0.78 \\
(0.67,0.89)\end{array}$ & $\begin{array}{c}0.71 \\
(0.67,0.74)\end{array}$ \\
\hline Non-dominant arm $\mathrm{R}_{0} /$ non-dominant leg $\mathrm{R}_{0}$ & 1.211 & $\begin{array}{c}0.78 \\
(0.65,0.89)\end{array}$ & $\begin{array}{c}0.64 \\
(0.60,0.67)\end{array}$ \\
\hline Dominant arm $\mathrm{R}_{\mathrm{i}}: \mathrm{R}_{0} /$ dominant leg $\mathrm{R}_{\mathrm{i}}: \mathrm{R}_{0}$ & 0.903 & $\begin{array}{c}0.72 \\
(0.59,0.85)\end{array}$ & $\begin{array}{c}0.63 \\
(0.60,0.67)\end{array}$ \\
\hline Non-dominant arm $\mathrm{R}_{\mathrm{i}}: \mathrm{R}_{0} /$ non-dominant leg $\mathrm{R}_{\mathrm{i}}: \mathrm{R}_{0}$ & 0.905 & $\begin{array}{c}0.80 \\
(0.65,0.89)\end{array}$ & $\begin{array}{c}0.73 \\
(0.69,0.76)\end{array}$ \\
\hline Model adjusted for sex, age and body mass index & & & \\
\hline Dominant arm $\mathrm{R}_{0}$ / dominant leg $\mathrm{R}_{0}$ & 0.045 & $\begin{array}{c}0.87 \\
(0.76,0.96)\end{array}$ & $\begin{array}{c}0.83 \\
(0.80,0.86)\end{array}$ \\
\hline Non-dominant arm $\mathrm{R}_{0} /$ non-dominant leg $\mathrm{R}_{0}$ & 0.042 & $\begin{array}{c}0.89 \\
(0.78,0.99)\end{array}$ & $\begin{array}{c}0.83 \\
(0.80,0.85)\end{array}$ \\
\hline Dominant arm $\mathrm{R}_{\mathrm{i}}: \mathrm{R}_{0} /$ dominant leg $\mathrm{R}_{\mathrm{i}}: \mathrm{R}_{0}$ & 0.050 & $\begin{array}{c}0.83 \\
(0.72,0.93)\end{array}$ & $\begin{array}{c}0.80 \\
(0.76,0.82)\end{array}$ \\
\hline Non-dominant arm $\mathrm{R}_{\mathrm{i}}: \mathrm{R}_{0} /$ non-dominant leg $\mathrm{R}_{\mathrm{i}}: \mathrm{R}_{0}$ & 0.048 & $\begin{array}{c}0.87 \\
(0.76,0.96)\end{array}$ & $\begin{array}{c}0.84 \\
(0.81,0.86)\end{array}$ \\
\hline
\end{tabular}


Supplementary Table 1 . Sensitivity and specificity of normative data-derived cut-offs.

\begin{tabular}{lccc}
\hline Criteria & Cut-offs* & Sensitivity $(95 \% \mathrm{CI})$ & $\begin{array}{c}\text { Specificity }(95 \% \\
\text { CI })\end{array}$ \\
\hline Inter-leg $\mathrm{R}_{0}$ ratios & & & \\
\hline$\mu+0.5 \sigma$ & 1.041 & $0.45(0.31-0.60)$ & $0.79(0.67-0.87)$ \\
$\mu+0.75 \sigma$ & 1.059 & $0.29(0.17-0.43)$ & $0.91(0.82-0.97)$ \\
$\mu+1 \sigma$ & 1.077 & $0.22(0.12-0.37)$ & $0.96(0.88-0.99)$ \\
$\mu+1.5 \sigma$ & 1.113 & $0.20(0.10-0.34)$ & $1.00(0.95-1.00)$ \\
$\mu+2 \sigma$ & 1.149 & $0.14(0.06-0.27)$ & $1.00(0.95-1.00)$ \\
$\mu+2.5 \sigma$ & 1.185 & $0.14(0.06-0.27)$ & $1.00(0.95-1.00)$ \\
$\mu+3 \sigma$ & 1.221 & $0.12(0.05-0.25)$ & $1.00(0.95-1.00)$
\end{tabular}

\begin{tabular}{lccc}
\hline Arm-to-leg $\mathrm{R}_{0}$ ratios & & \\
\hline$\mu+0.5 \sigma$ & $1.209-1.252$ & $0.84(0.70-0.93)$ & $0.64(0.54-0.74)$ \\
$\mu+0.75 \sigma$ & $1.249-1.296$ & $0.80(0.66-0.90)$ & $0.72(0.62-0.81)$ \\
$\mu+1 \sigma$ & $1.289-1.339$ & $0.73(0.59-0.85)$ & $0.84(0.75-0.91)$ \\
$\mu+1.5 \sigma$ & $1.369-1.426$ & $0.59(0.44-0.73)$ & $0.91(0.83-0.96)$ \\
$\mu+2 \sigma$ & $1.449-1.513$ & $0.55(0.40-0.69)$ & $0.97(0.91-0.99)$ \\
$\mu+2.5 \sigma$ & $1.529-1.600$ & $0.49(0.34-0.64)$ & $0.99(0.94-1.00)$ \\
$\mu+3 \sigma$ & $1.609-1.687$ & $0.41(0.27-0.56)$ & $0.99(0.94-1.00)$ \\
\hline Arm-to-leg $\mathrm{R}_{\mathrm{i}}: \mathrm{R}_{0}$ ratios & & \\
\hline$\mu+0.5 \sigma$ & $1.301-1.421$ & $0.06(0.01-0.17)$ & $0.62(0.51-0.72)$ \\
$\mu+0.75 \sigma$ & $1.392-1.534$ & $0.06(0.01-0.17)$ & $0.68(0.57-0.77)$ \\
$\mu+1 \sigma$ & $1.484-1.647$ & $0.04(0.00-0.14)$ & $0.70(0.59-0.79)$ \\
$\mu+1.5 \sigma$ & $1.668-1.874$ & $0.04(0.00-0.14)$ & $0.77(0.67-0.85)$
\end{tabular}




\begin{tabular}{llll}
$\mu+2 \sigma$ & $1.851-2.100$ & $0.04(0.00-0.14)$ & $0.86(0.77-0.92)$ \\
$\mu+2.5 \sigma$ & $2.035-2.327$ & $0.02(0.00-0.11)$ & $0.93(0.86-0.98)$ \\
$\mu+3 \sigma$ & $2.218-2.553$ & $0.02(0.00-0.11)$ & $0.98(0.92-1.00)$ \\
\hline Arm-to-leg $R_{0}$ ratios, stratified by sex & \\
\hline$\mu+0.5 \sigma$ & $1.095-1.298$ & $0.80(0.66-0.90)$ & $0.61(0.50-0.71)$ \\
$\mu+0.75 \sigma$ & $1.123-1.339$ & $0.71(0.57-0.83)$ & $0.73(0.63-0.82)$ \\
$\mu+1 \sigma$ & $1.151-1.381$ & $0.65(0.50-0.78)$ & $0.81(0.71-0.89)$ \\
$\mu+1.5 \sigma$ & $1.208-1.465$ & $0.59(0.44-0.73)$ & $0.90(0.82-0.95)$ \\
$\mu+2 \sigma$ & $1.264-1.548$ & $0.55(0.40-0.69)$ & $0.97(0.91-0.99)$ \\
$\mu+2.5 \sigma$ & $1.321-1.632$ & $0.51(0.36-0.66)$ & $0.99(0.94-1.00)$ \\
$\mu+3 \sigma$ & $1.377-1.715$ & $0.39(0.25-0.54)$ & $0.99(0.94-1.00)$
\end{tabular}

Arm-to-leg $\mathrm{R}_{0}$ ratios, stratified by sex and age

\begin{tabular}{llll}
\hline$\mu+0.5 \sigma$ & $1.045-1.323$ & $0.75(0.60-0.86)$ & $0.66(0.55-0.75)$ \\
$\mu+0.75 \sigma$ & $1.068-1.372$ & $0.69(0.54-0.81)$ & $0.77(0.67-0.85)$ \\
$\mu+1 \sigma$ & $1.091-1.421$ & $0.65(0.49-0.78)$ & $0.80(0.70-0.88)$ \\
$\mu+1.5 \sigma$ & $1.137-1.519$ & $0.50(0.35-0.65)$ & $0.88(0.79-0.94)$ \\
$\mu+2 \sigma$ & $1.182-1.617$ & $0.48(0.33-0.63)$ & $0.93(0.86-0.98)$ \\
$\mu+2.5 \sigma$ & $1.229-1.715$ & $0.42(0.28-0.57)$ & $0.94(0.88-0.98)$ \\
$\mu+3 \sigma$ & $1.275-1.813$ & $0.33(0.20-0.48)$ & $0.97(0.91-0.99)$
\end{tabular}

*Cut-offs vary based on dominant or non-dominant side of the body (non-stratified cut-offs), sex and age (stratified cut-offs). 